\title{
A Kind of Ant Colony Algorithm with Route Evaluation
}

\author{
Fang Zhao*
}

Department of Computer and Information Engineering, Xinxiang University, Xinxiang City, 453003, China

\begin{abstract}
Ant algorithm is a novel meta-heuristic optimization algorithm, but at the same time there are some disadvantages. This paper proposed an ant colony algorithm with route evaluation based on the basic ant colony algorithm. After all ant complete the route search once, the current search situation can be evaluated according to an improved formula of standard deviation, then to decide to update global pheromone or not. At the same time, via the function of interval compression, the pheromone of all routes is compressed to adjust the searching probability of ants to each city. Experimental results show that this improved algorithm has more excellent presentation on convergence, searching optimization, and stability.
\end{abstract}

Keywords: Pheromone, ant colony algorithm, route evaluation, pheromone interval, compression circle.

\section{INTRODUCTION}

Ant algorithm is a kind of meta-heuristic algorithm proposed by Marco Dorigo, et al. [1,2] scholars in the inspiration by real ants foraging behavior, which is not only successfully used to solve TSP problem [3, 4], and in solving combinatorial optimization problems [3] such as scheduling [4], graph coloring problem [5] etc., good results also achieved. Ant algorithm in solving combinatorial optimization problems which can be presented by available diagrams has the potential superiority, therefore this attracts the attention of many researchers and in this study, and some improved algorithms [6-8] are put forward.

By pheromones the ants decide the next mobile site. With the background of discussing the TSP problem, they have the following characteristics:

- When moving from the city to the city, the pheromone is placed on edge.

- Through the transition probability (the function of distance between the cities and sum of pheromones on the connection edge) to select the next city to go to.

- In order to make artificial ants to obtain legal path, unless a path is complete, otherwise, the cities which have been asked are not allowed to visit again.

Ant algorithm has been successfully applied in many different optimization problems, but it has the disadvantages such as easy prematurity, slow convergence speed in the search process. Aiming to these shortcomings of ant colony algorithm, this paper proposes an ant colony algorithm with path evaluation; this algorithm can effectively overcome the shortcomings in the search process, and has the better search ability and better stability.

\section{ANT COLONY ALGORITHMS}

\subsection{The TSP Problem}

The TSP problem of $n$ cities is to find a closed loop path with the shortest length, which has only visited each city once. If $d_{i j}$ presents the distance between city $i$ and city $j$, $\tau_{i j}(t)$ is the information amount at the moment $t$ on the path $(i, j), n$ presents the TSP size, $m$ is the total number of ant colony; $\operatorname{tabu}_{k}(k=1,2, \ldots, m)$ presents the taboo list that ant $k$ currently walks through the cities, when all $n$ cities join into $t a b u_{k}$, then ant $k$ completes a search, the path experienced by ant $k$ is a solution of the TSP problem.

\subsection{The Resolving of TSP Based on an Ant Colony Algo- rithm}

The $m$ ants are randomly placed into $n$ cities, at the same time, the first element of each ant tabu list is set to its current city, at this time the pheromone of each path is equal, which is set to a constant $\tau_{0}$, then each ant independently selects the next city. The build process of path is as follows.

At the moment $t$, the transition probability of ant $k$ moving from city $i$ to city $j$ is:

$p_{i j}^{k}(t)=\left\{\begin{array}{c}\frac{\left[\tau_{i j}(t)\right]^{\alpha} \cdot\left[\eta_{i j}(t)\right]^{\beta}}{\sum_{\text {seallowed }_{k}}\left[\tau_{i s}(t)\right]^{\alpha} \cdot\left[\eta_{i s}(t)\right]^{\beta}}, \text { if jeallowed } k \\ 0, \text { otherwise }\end{array}\right.$

In the expression, allowed $_{k} \bullet\{1,2, \ldots, n\}-$ tabu $_{k}$ represents the next city that ant $k$ is allowed to select; $\alpha$ is the infor- 
mation heuristic factor; $\beta$ is the expecting heuristic factor; $\eta_{i j}(t)$ is the heuristic function. The pheromone updates as follows.

When all the ants complete a travel once, the pheromone on each path updates according to expressions (2), (3).

$\tau_{i j}(t+n)=(1-\rho) \tau_{i j}(t)+\Delta \tau_{i j}(t)$

$\Delta \tau_{i j}(t)=\sum_{k=1}^{m} \Delta \tau_{i j}^{k}(t)$

$\Delta \tau_{i j}^{k}(t)=\left\{\begin{array}{cl}\frac{Q}{L_{k}}, & \text { if ant } k \text { passes path }(i, j) \text { in this travel } \\ 0 & \quad \text { otherwise }\end{array}\right.$

In the expression, $\rho$ represents pheromone volatilization coefficient, $1-\rho$ represents pheromone residual factor; $\Delta \tau_{i j}(t)$ represents the increment of the pheromone in this loop on the path $(i, j), \Delta \tau_{i j}^{k}(t)$ represents the information amount of the $k$-th ant in the loop left on the path $(i, j), Q$ is a positive constant, $L_{k}$ represents the path length of $k_{-t h}$ passed through the travel.

\section{THE ANT COLONY ALGORITHM WITH ROUTE} EVALUTION (ACARE)

\subsection{The Solving of TSP Based on ACARE}

The path construction:

The ant $k$ Located in the city $i$, according to the rules of pseudo-random proportional the city $j$ is selected as the next city. The rule is given by the following expression:

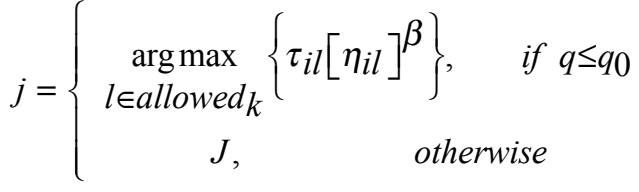

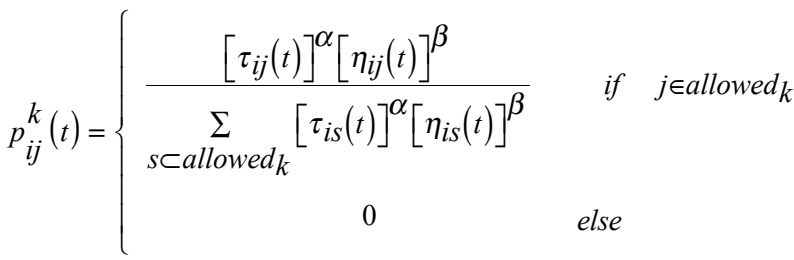

In the expression, $q$ is a random variable uniformly distributed in the range of $[0,1], q_{0}\left(0 \leq q_{0} \leq 1\right)$ is a parameter, $J$ is a random variable produced according to the probability distribution of given (6).

Global pheromone update:
Only the current optimal path can be done the global pheromone update, the rules are as follows:

$\tau_{i j}=(1-\rho) \tau_{i j}+\rho \Delta \tau_{i j}^{b s} \forall(i, j) \in T^{b s}$

$\Delta \tau_{i j}^{b s}=1 / C^{b s}$

Local pheromone update:

After an ant finds out a path, the current path can be done locally pheromone update, the update rules are as follows:

$\tau_{i j}=(1-\rho) \tau_{i j}+\xi \tau_{0}$

In the expression, $\xi$ and $\tau_{0}$ are two parameters, $\xi$ meets $0<\xi<1, \tau_{0}$ is the initial value of pheromone volume.

ACARE is used to do the improvement in the following two aspects:

(1) According to the improved variance calculation formulas to evaluate current path, it is used to determine if to implement a global pheromone update.

When all the ants complete a route search, according to the standard variance formula (10) the variance can be calculated.

$F=\sqrt{\frac{\sum_{1}^{m}\left(x_{i}-\bar{x}\right)^{2}}{m}}$

Thereinto, $m$ represents the ants number, $x_{\mathrm{i}}$ represents the path length searched by the $i$-th ant in the circular search, $\bar{x}$ represents the average value of path length that $m$ ants found.

For different sizes of TSP problem, $x_{\mathrm{i}}$ is not the same, the calculated variance may be great, now formula (10) is modified as follows:

$F=\frac{\sqrt{\sum_{1}^{m}\left(x_{\mathrm{i}}-\bar{x}\right)^{2}}}{\text { NNTour }}$

Hereinto, NNTour represents the route length according to the greed rules.

According to the standard variance formula, when the data distribution is closer to the average, the standard deviation is smaller; the further away is from the average data distribution, the bigger is the standard deviation. Therefore, if $F$ is larger, which means the length difference of $m$ paths that $m$ ants find is larger, that is, the $m$ paths differences are also bigger, the search scope in the loop search for ant is larger; If $F$ is small, which means the length difference of $m$ paths that ants find is lesser, that is, the $m$ paths differences are also small, the search scope in the loop search for ant is smaller. 
Therefore, at the early stage of the search, if the calculated variance is small, the search range is small, in order to have larger search range, at the time of pheromone update, only local pheromone can update without a global update, it won't make the pheromone on current optimal path to increase, increasing the next cycle ant chooses the probability of this path to make the algorithm lost in local search, and lead to premature phenomenon; if the calculated variance is bigger, the search range is larger, which are regarded as normal, global pheromone updating and local pheromone updating are performed, it is as same as in the later stage of search.

Here, the search is divided into three stages, and parameters $a$ and $b, a>b$ are introduced, the parameters $a$ and $b$ are called the block parameters. In the early stage, comparing $a$ and variance $F$, only when the calculated variance $F>a$, global update can be done. In mid-term stage, comparing $b$ with variance $F$, only when the calculated variance $F>b$, global update can be done; in the late stage, global pheromone updating and local pheromone updating are performed.

(2) Adopt the range compression method to adjust the pheromone on all trails

Definition 3.1 pheromone interval: at the same time, the pheromone on the paths between two cities distributes in an interval, this range is called pheromone interval [9].

In the searching process of ants, if in a certain period of time there is no more superior solution, it may be premature, the pheromone in some local path is significantly higher than that on the other paths, which makes subsequent ants to choose a local path with higher probability, at this time the pheromone range is larger. The compression function compresses the pheromone interval, the compressed pheromone is distributed in a smaller range, which makes the gap of pheromone on the paths smaller, adjusts the selection probability in the search process of ants, and increases the search probability of other paths.

Definition 3.2 compression cycles: every once in a while to compress the pheromones on all paths, this period of time is called the compression cycle [10], it is marked as $T_{y s}$.

The compression cycle cannot be too small. Pheromones compression adjusts the selection probability of ants to each city. According to the compressed pheromone on the paths, over a period of time a better solution may be found after the search, if the compression cycle is too small, the opportunity may be destroyed to get a better solution.

There are two kinds of compression strategy: linear subsection compression and nonlinear compression [11].

Strategy 1: linear subsection compression

The range compression function is:

$$
\begin{aligned}
& y=A x \quad A \in(0,1) \\
& A=\left\{\begin{array}{lll}
q_{1}-w & \text { if } & \tau_{i j}<\tau \\
q_{2}+w & \text { if } & \tau_{i j} \geq \tau
\end{array}\right.
\end{aligned}
$$

In the expression, $q_{1}, q_{2} \in(0,1), \quad w$ is a perturbation parameter, $w \in[0,0.05), \tau_{i j}$ is the pheromone on the path $(i, j)$, $\tau$ is a value in the pheromone interval.

Strategy 2: nonlinear compression (secondary compression)

The range compression function is:

$y=a x^{2}+b x+c$

In the expression, $a, b, c$ are three constant parameters.

\subsection{The Implementation of ACARE Program}

Begin

Initialize the parameters, $n c y c l e=0, t_{a b u_{k}}=0$;

While(ncycle $<=$ MAXcycle)

\{

For(antnumber $=0$; antnumber $<=M$; antnumber ++ ) $\left\{\right.$ while $\left(\right.$ tabu $_{k}<$ citynumber $)$

$\{$ according expression (5) to select the next city; $t_{a b u_{k}++;}$

if $\left(\right.$ tabu $_{k}=$ citynumber $)$ the ant return

back to the start city;

according to expression (9) to update

current path pheromone; comparing

with current route;

\}

Confirm the optimal route of this circle; and calculate the variance $F$;

if (early stage)

$\{$ if $(F>a)$ according to expression (7) to globally update the pheromone of current optimal route;

if (middle stage)

$\{$ if $(F>b)$ according to expression (7) to globally update the pheromone of current optimal

route; \}

else to globally update the pheromone of current optimal route;

if (there is no better solution in a period of time)

$\{$ For the pheromone distribution interval of all routes according to the expression (12) or

(14) to do range compression; \}

\}

Output the result

End

\subsection{The Simulation Experiments}

\subsubsection{Parameter Selection}

Ant number is 1.5 times to the number of city, the number of iterations is decided according the solved problem size, 


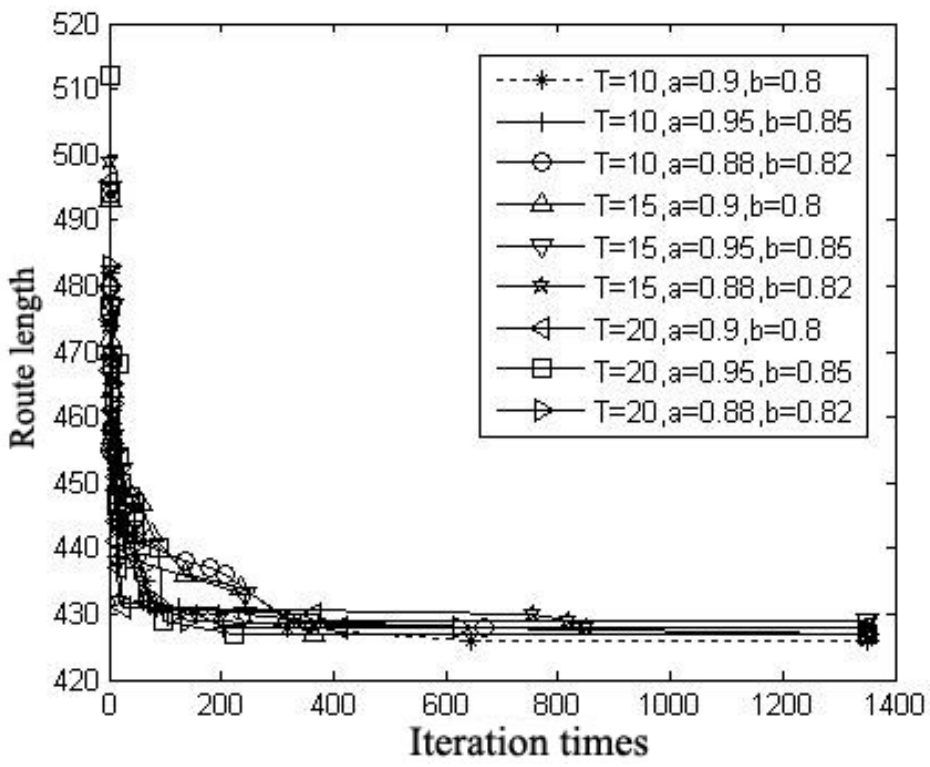

Fig. (1). Nonlinear compression figure, using one segment dividing method, the compression cycle and staging parameters with mutual collocation.

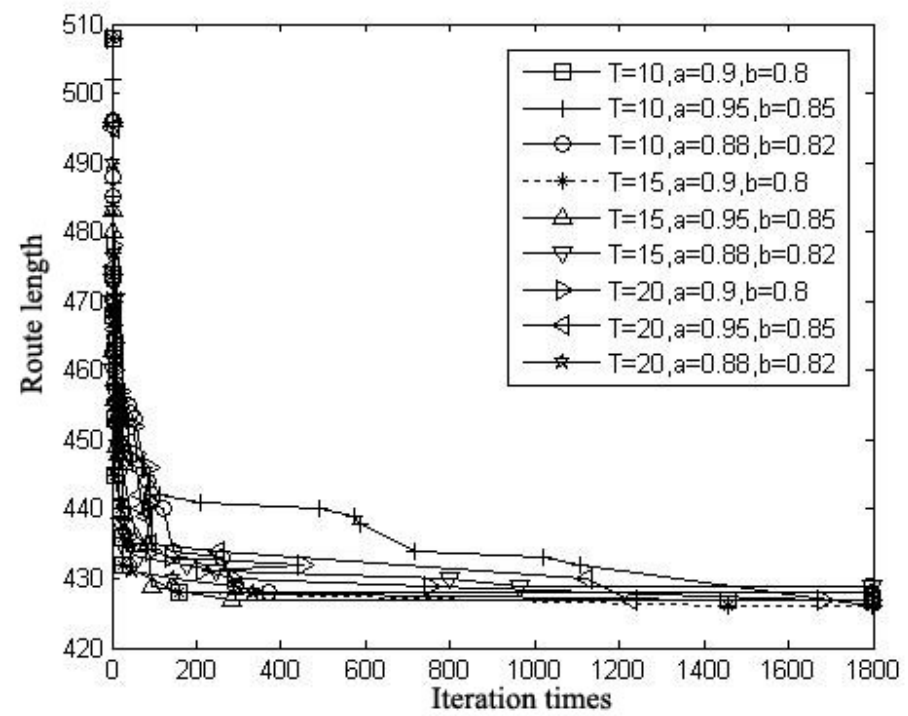

Fig. (2). Nonlinear compression convergence figure, using two segments dividing method, the compression cycle and staging parameters with mutual collocation.

compression cycle is selected three ways: $T_{y s}=10,15,20$;

Through the experimental statistical analysis of variance, block parameters select $a=0.88, \quad b=0.82 ; a=0.9, b=0.8$; and $a=0.95 b=0.85$ three groups, the dividing methods of three stages of the ant search are: method 1: $T_{1}=\frac{1}{3} T \quad T_{2}=\frac{2}{3} T$; method 2: $T_{1}=\frac{1}{5} T \quad T_{2}=\frac{3}{5} T$, the simulation experiments are done based on these parameters (match each other). The rest of the parameters are as follows:

Strategy 1: using linear subsection compression

If the value of $A$ in expression (12) is too small, the pheromone range compression is bigger, the pheromone on

each edge is very close, the change of the ant selecting probability to next city is too large, which will affect the convergence of the algorithm; If the value of $A$ is too large, it is not very obvious to adjust the ant search probability. Therefore, the value of $A$ is taken near the golden section ratio $[0,1]$, in expression (13) $q_{1}$ is taken $0.7, q_{2}$ is taken $0.6, w$ is a disturbance of random umber in $[0,0.05), \tau$ is the midpoint of pheromone interval.

Strategy 2: nonlinear compression (secondary compression). 


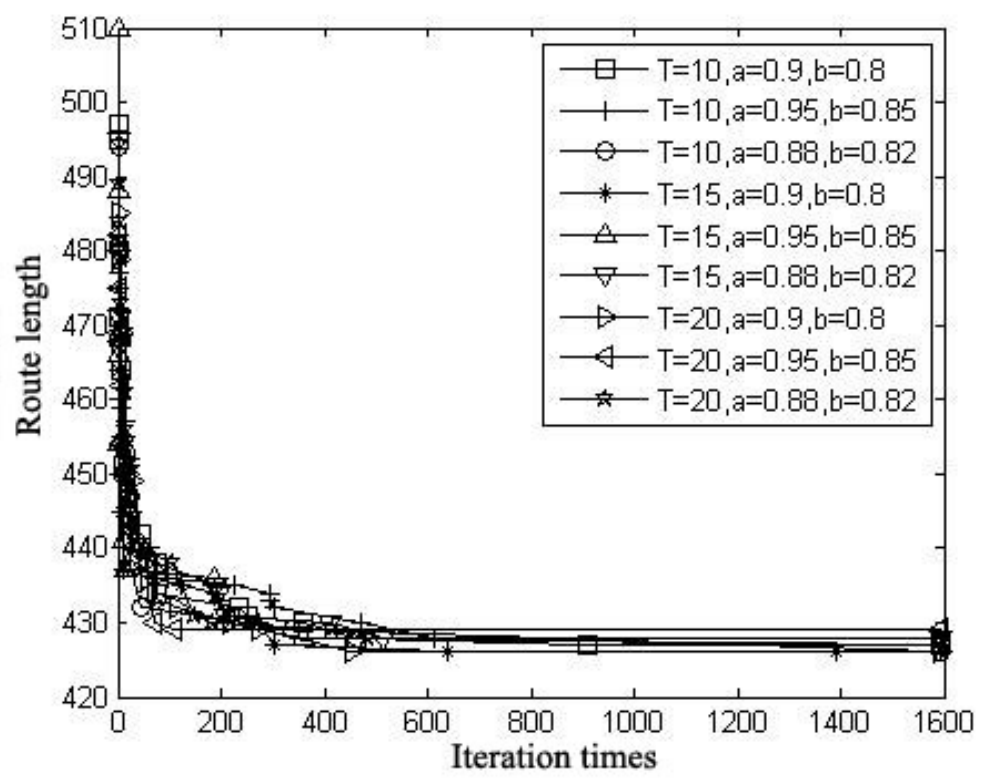

Fig. (3). Linear compression convergence figure, using a segment dividing method, the compression cycle and staging parameters with mutual collocation.

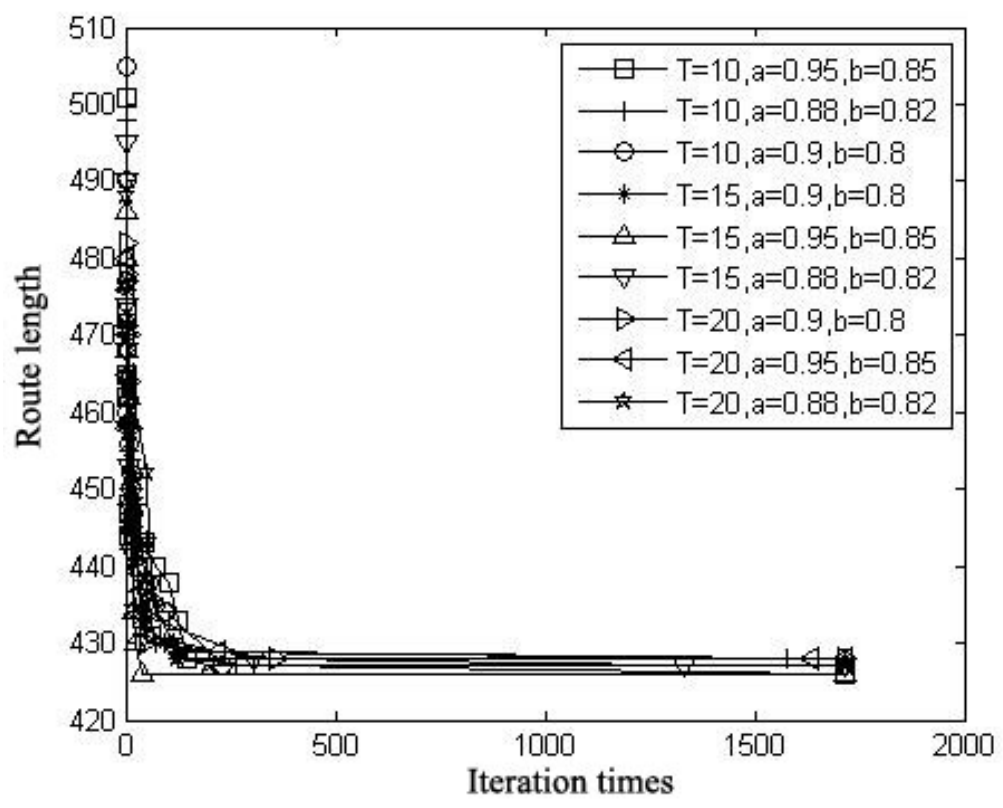

Fig. (4). Linear compression convergence figure, using two segments dividing method, the compression cycle and staging parameters with mutual collocation.

Through the statistical analysis to the pheromone on each edge, with the aid of Matlab tool, in the expression (14), it is obtained that $a=-1116.7, b=15, c=0$.

\subsubsection{Experimental Results}

In Fig. (1-4), eil51 problem is given, and the iteration number is 2000 times, the convergence condition figures, such as a phase dividing method, compression cycle and segmentation parameter match with each other, presents that the improved ACARE algorithm has better convergence.
In Table 1, aiming to eil51, eil101, ch150 three problems, the AS, ACS and ACARE three algorithms run 10 consecutive tests to obtain the statistical data.

From Table 2, the improved ACARE has better ability to find a better solution. The proximity between found optimal solution and known optimal solution, and the statistical result's average of continuous running for ten times, are superior to AS algorithm and ACS algorithm; From the value of statistical standard variance, ACARE algorithm is better than AS algorithm and ACS algorithm at the stability. 
Table 1. The basic parameters of ACS and ACARE.

\begin{tabular}{|c|c|c|c|c|c|c|c|c|c|c|}
\hline \multicolumn{3}{|c|}{ AS } & \multicolumn{9}{c|}{ ACS } & \multicolumn{3}{c|}{ ACARE } \\
\hline \hline$\alpha$ & $\beta$ & $\rho$ & $\alpha$ & $\beta$ & $\rho$ & $\xi$ & $\alpha$ & $\beta$ & $\rho$ & $\xi$ \\
\hline 1 & 2 & 0.1 & 1 & 2 & 0.1 & 0.1 & 1 & 2 & 0.1 & 0.15 \\
\hline
\end{tabular}

Table 2. AS, ACS, and ACARE algorithms testing data aiming to eil51, eil101, and ch150.

\begin{tabular}{|c|c|c|c|c|c|c|c|}
\hline TSP & & Algorithm & & Best & Worst & Avg & Stdv \\
\hline \multirow{6}{*}{ Eil51 } & & AS & & 430 & 439 & 435.9 & 2.385 \\
\hline & & $\mathrm{ACS}$ & & 426 & 424 & 430.0 & 3.346 \\
\hline & \multirow{4}{*}{ ACARE } & \multirow{2}{*}{ Method 1} & $\begin{array}{c}\text { Strategy } \\
1\end{array}$ & 426 & 429 & 427.9 & 0.943 \\
\hline & & & $\begin{array}{c}\text { Strategy } \\
2\end{array}$ & 426 & 430 & 427.6 & 1.019 \\
\hline & & \multirow{2}{*}{ Method 2} & $\begin{array}{c}\text { Strategy } \\
1\end{array}$ & 426 & 430 & 427.8 & 1.249 \\
\hline & & & $\begin{array}{c}\text { Strategy } \\
2\end{array}$ & 426 & 429 & 427.7 & 1.135 \\
\hline \multirow{6}{*}{ Eil101 } & \multicolumn{3}{|c|}{ AS } & 642 & 655 & 647.9 & 3.590 \\
\hline & \multicolumn{3}{|c|}{$\mathrm{ACS}$} & 636 & 647 & 641.5 & 3.528 \\
\hline & \multirow{4}{*}{ ACARE } & \multirow{2}{*}{ Method 1} & $\begin{array}{c}\text { Strategy } \\
1\end{array}$ & 630 & 637 & 633.3 & 1.846 \\
\hline & & & $\begin{array}{c}\text { Strategy } \\
2\end{array}$ & 631 & 640 & 635.6 & 1.624 \\
\hline & & & $\begin{array}{c}\text { Strategy } \\
1\end{array}$ & 630 & 639 & 634.4 & 1.907 \\
\hline & & & $\begin{array}{c}\text { Strategy } \\
2\end{array}$ & 631 & 638 & 633.8 & 1.939 \\
\hline \multirow{6}{*}{ Ch150 } & & AS & & 7014 & 7491 & 7202. & 107.7 \\
\hline & & ACS & & 6826 & 7094 & 7007. & 53.36 \\
\hline & \multirow{4}{*}{ ACARE } & \multirow{2}{*}{ Method 1} & $\begin{array}{c}\text { Strategy } \\
1\end{array}$ & 6618 & 6729 & 6659.4 & 25.5625 \\
\hline & & & $\begin{array}{c}\text { Strategy } \\
2\end{array}$ & 6623 & 6751 & 6669.8 & 28.6672 \\
\hline & & \multirow{2}{*}{ Method 2} & $\begin{array}{c}\text { Strategy } \\
1\end{array}$ & 6601 & 6737 & 6650.8 & 31.878 \\
\hline & & & $\begin{array}{c}\text { Strategy } \\
2\end{array}$ & 6641 & 6774 & 6687.5 & 30.2538 \\
\hline
\end{tabular}




\section{CONCLUSION}

The ant colony algorithm with path evaluation is proposed in this paper, has significantly improved at the convergence, stability, and searching the optimal solution, which can effectively overcome the problems in the basic ant colony algorithm such as easy to be premature and trapping in local optimal solution. Through simulation experiments, the performance of an ant colony algorithm with path evaluation has better performance than the basic ant colony algorithm.

\section{CONFLICT OF INTEREST}

The author confirms that this article content has no conflict of interest.

\section{ACKNOWLEDGEMENTS}

Declared none.

\section{REFERENCES}

C. M. Dorigo, and V. Maniezzo, "Distributed optimization by ant colonies", In Proceedings of the First European Conference on Artificial Life, pp.134-142, 1992.
[2] M. Dorigo,V. Maniezzo and A. Colorni, "The ant system: Optimization by a colony of cooperating agents", IEEE Transactions on System, Man, and Cybernetics, Part B: Cybernetics, vol. 26 pp. 29-41, 1996

[3] M. Dorigo, L. M. Gambardella, "Ant colony system: a cooperative learning approach to the traveling salesman problem", IEEE Transactions on Evolutionary Computation, vol. 1, pp. 53-66, 1997.

[4] A. Colorni, M. Dorigo, and V. Maniezzo, "Ant colonies for jobshop scheduling", Belgian Journal of Operations Research, Statistics and Computer Science (JORBEL), vol. 34, pp. 39-54, 1994.

[5] D. Costa and A. Hertz, "Ants can color graphs", Journal of the Operational Research Society, vol. 48, pp. 295-305, 1997.

[6] H. Duan and D. Wang, "A fast global optimization of the improved ant colony algorithm and simulation", Information and control, vol. 33, pp. 241-244, 2004.

[7] H. Duan and G. Ma, "An improved ant colony algorithm to solve the continuous space optimization problem", Journal of System Simulation, vol. 19, pp. 974-977, 2007.

[8] X. Zhang and L. Tang, "A ACO\&SS algorithm design to solve TSP problem", Control and Decision, vol. 23, pp. 762-766, 2008.

[9] L. Jiao, H. Du, and F. Liu, "The immune optimization calculation, learning and recognition", Beijing: Science Press, pp. 92-98, 2006.

[10] T. H. Guo and Z. Michalewicz, "Inver-over Operator for the TSP", Proceedings of the 5th Parallel Problem Solving From Nature. Berlin: Springer, pp. 803-812, 2009.

[11] L. Wang, J. Pan and L. Jiao, "Immunization programs", Journal of Computers, vol. 23, pp. 806-812, 2000.

(C) Fang Zhao; Licensee Bentham Open.

This is an open access article licensed under the terms of the Creative Commons Attribution Non-Commercial License (http://creativecommons.org/licenses/by-nc/3.0/) which permits unrestricted, non-commercial use, distribution and reproduction in any medium, provided the work is properly cited. 